\title{
Local Lymph Node Assay: How Testing Laboratories Apply OECD TG 429 for REACH Purposes
}

\author{
Costanza Rovida
}

CAAT-Europe, University of Konstanz, Germany

\begin{abstract}
Summary
The Local Lymph Node Assay (LLNA) is the official method for assessing the allergic contact dermatitis potential of chemicals for the purposes of REACH regulation. The LLNA went through a validation process that allowed the delineation of a robust protocol for performing new tests. The OECD accepted this method in 2002 and published OECD TG 429. The European Chemical Agency (ECHA) recently published data that were submitted in the registration dossiers of chemicals. This database was analysed to determine how testing laboratories apply OECD TG 429. This analysis comes after a detailed analysis of four full study reports that were also prepared for REACH purposes. Although the majority of the tests are fully compliant with OECD TG 429, some showed major deviations, and a number of others used more animals than necessary. This suggests that in vivo tests need to be planned more carefully and consciously to obtain meaningful results with the minimum animal number necessary.
\end{abstract}

Keywords: LLNA, REACH, standardisation, GLP compliance

\section{Introduction}

\subsection{The situation before REACH}

The allergic contact dermatitis potential of chemicals generally is assessed in vivo using three methods described in two OECD Test Guidelines (TG):

- OECD TG 406 on the Guinea Pig Maximisation Test (GPMT) and the Buehler Assay

- OECD TG 429 on the Local Lymph Node Assay (LLNA)

Compared to the GPMT and the Buehler assay, the LLNA requires fewer animals (reduction), and both the allergic reaction and the suffering of the animals during treatment are less severe (refinement), thus it is considered an alternative method in the sense of the $3 \mathrm{Rs}$ (reduction, refinement, replacement). It is still an in vivo method, however, so the most import $\mathrm{R}$ (replacement) has not yet been achieved and therefore the use of the LLNA, like that of any other in vivo methods, is recommended only when absolutely necessary. The goal of this paper is to assess how the LLNA is applied for the purposes of REACH (Registration, Evaluation, Authorisation and restriction of Chemicals), the regulation for the management of chemical substances in the European Union (EC, 2006). The analysis of a number of published LLNA studies reveals how little attention is often given to the planning and execution of a new in vivo study, as demonstrated by protocols that do not completely follow the guidelines and do not ensure the use of as few animals as possible and by final reports that do not contain all relevant information.

The LLNA is the only validated in vivo method. It was developed in the early 1990s (Kimber and Basketter, 1992), and went through an extensive validation process. In 1999, the validation report (NTP, 1999) was published on the website of the US National Institutes of Health (NIH). Three years later, the OECD accepted it and issued OECD guideline TG 429 in July 2002. Recently (July 2010) a revision of guideline 429 was published which introduces minor changes to the main protocol.

The LLNA has many advantages over the GPMT and the Buehler test. From a scientific point of view, the fact that it has been validated means that it has been studied extensively and the protocol is optimised for best performance. Moreover, the LLNA provides a dose response assessment that allows some considerations about the potency of the tested substance rather than a simple yes/no answer. It is also faster, taking only 6 days, compared to tests on guinea pigs, which take at least 24 days. The LLNA does not require elicitation of dermal hypersensitivity reactions, and the application of the substance onto animal skin is done without an adjuvant; therefore the pain and distress caused to the animal is very much reduced.

Although both the GPMT and the Buehler test have considerable credibility, they often return either false positive or false

Received February 22, 2011; accepted in revised form April 12, 2011. 
negative results, as the test interpretation requires experience and expertise (Basketter et al., 2009). The LLNA takes advantage of a much more standardised procedure, in which the final outcome relies on an instrumental measurement that is much less prone to subjective interpretation. The use of the EC3 value (effective concentration inducing a 3-fold increase in proliferation of lymph node cells) for categorisation of allergic contact dermatitis potential in the LLNA is not yet fully accepted by regulators, even if evaluation is well advanced. Cockshott et al. (2006) performed a detailed assessment of the LLNA from a regulatory perspective, confirming its suitability in comparison with other in vivo test methods. The LLNA has some limitations such as the probability of a false positive outcome from strong irritants (Basketter et al., 2009; Basketter and Kimber, 2009). At least these drawbacks are well known, which is not the case for other in vivo studies as these have not undergone validation.

Even though the advantages are evident, in practice OECD TG 429 has not replaced OECD TG 406, and the Buehler test and the GPMT are still more frequently applied for regulatory purposes than the LLNA. This is probably due to historical reasons: testing labs tend to stick to methods that they have always used if they are not forced to change. Also, the need to use radioactive material in the original LLNA protocol and the associated measures may be a deterrent. In fact, few testing laboratories have permission to work with ${ }^{3} \mathrm{H}$-methyl thymidine. In more recent years, modified versions of the LLNA that do not require radioactive material have been developed and validated and now, two new guidelines, the OECD TG 442a and 442b, fully endorse those modified versions. Shortly, these methods measure the lymphocyte proliferation by quantifying the adenosine triphosphate content via bio-luminescence and 5-bromo-2-deoxyuridine (BrdU) in an ELISA-based test system respectively.

This was the situation until December 30, 2006 when REACH was published (EC, 2006).

\subsection{REACH and LLNA}

The main purpose of REACH is to evaluate the safety of all substances circulating in the EU. For this, a chemical registration dossier, which includes analytical characterisation, physico-chemical properties, environmental fate, ecotoxicological profile, toxicological profile, intended use and disposal of the substance and risk management measures, is required for every chemical. For substances marketed in quantities greater than 10 tons/year, the registration dossier must also contain the Chemical Safety Report (CSR), which is the result of an in depth Chemical Safety Assessment (CSA).

The required datasets and the deadline for submission of the dossier depends on the total quantity of chemical that is handled in a year by each company in the EU. This is described in Annexes VII through X of REACH. Skin sensitisation is an endpoint detailed in Annex VII which is mandatory for all substances manufactured or imported in quantities of one ton or more per year.

The precise wording on skin sensitisation in this REACH Annex is:

"COLUMN 1: STANDARD INFORMATION REQUIRED

8.3. Skin sensitisation - The assessment of this endpoint shall comprise the following consecutive steps:

(1) an assessment of the available human, animal and alternative data,

(2) In vivo testing.

COLUMN 2: SPECIFIC RULES FOR ADAPTATION FROM COLUMN 1

8.3. Step 2 does not need to be conducted if:

- the available information indicates that the substance should be classified for skin sensitisation or corrosivity, or

- the substance is a strong acid $(\mathrm{pH} \leq 2.0)$ or base $(\mathrm{pH} \geq 11.5)$, or

- the substance is flammable in air at room temperature.

The murine Local Lymph Node Assay (LLNA) is the firstchoice method for in vivo testing. Only in exceptional circumstances should another test be used. Justification for the use of another test shall be provided."

Those statements appear self-explanatory. First of all, it must be noted that the LLNA is indicated as the first choice for new tests. Column 2 also reinforces that in vivo testing is required only when no other data is available. Column 2 provides details about when the test can be waived due to technical reasons. In addition to this, Annex XI offers the possibility to use alternative approaches, such as read-across from similar substances, computer modeling and in vitro testing, provided that a robust scientific justification is available. Non validated methods can be applied in a weight-of-evidence approach, or appropriate integrated testing strategies can be built to reach a robust conclusion with fewer or no new in vivo tests.

The whole registration process is managed by ECHA located in Helsinki. The ECHA website (http://echa.europa.eu) provides guidance on the preparation of the registration dossier.

Many other Regulations and Directives are related to REACH. Regulation 440/2008 (EC, 2008a) lays down test methods pursuant to REACH. The LLNA is described in Chapter B.42 of this regulation in the exact words of OECD guideline 429 (2002 version). Regulation 440/2008 also includes a section endorsing guinea pig in vivo tests as previously described in OECD TG 406. In fact, section B.6 describes both the Guinea Pig Maximisation Test and the Buehler Test. The acceptability of all three in vivo methods is well described in the correspondent guideline published by ECHA, i.e. "Guidance on information requirements and chemical safety assessment Chapter R.7a: Endpoint-specific guidance" (ECHA, 2008). This guideline also states that any OECD method can be used for registration, even if it is not mentioned in Regulation $440 / 2008$, as is the case for the modified versions of the LLNA (OECD TG 442a and 442b).

The wording of the regulation indicates that the LLNA is the method of choice for new tests required for REACH purposes. The option to use integrated testing strategies or in vitro methods is rarely applied, as appears also from the preliminary analysis of the submitted dossiers presented in this paper. On the other hand, existing data must be exploited as much as possible, through the use of existing information on a specific substance or through read-across, i.e. by transposing results from similar substances. These options are accepted in the registration dossier when backed by robust scientific justification (ECHA, 2008). 
The registration dossier is prepared using IUCLID 5, a specific software available free of charge on the ECHA website (http://IUCLID.echa.europa.eu). This software is quite complex and requires that all study reports be retyped into the respective window. This forces testing laboratories to provide rich detail for all new experiments and reporting is more standardised. Regarding the LLNA and all other toxicological tests, ECHA Practical Guide 3 is the most relevant, as it addresses: "How to report robust study summaries". Chapter 5.2.3 of this technical guideline is about skin sensitisation (ECHA Practical Guide 3). It lists the minimum set of data that should be provided, including all parameters that are considered in this paper. The data of some of the submitted IUCLID 5 files are now publicly available on the ECHA website (http://apps.echa.europa.eu/registered/registered-sub.aspx).

The final assessment of the $\mathrm{REACH}$ registration dossier should lead to a decision on whether the substance is or is not a skin sensitiser. If it is a sensitiser, according to Regulation 1272/2008 on CLP (Classification, Labelling and Packaging), the chemical is classified as H317, a skin sensitiser (EC, 2008b). Classification is just a yes/no decision, and it does not require further assessment regarding the potency, such as classification of sensitisers as weak, moderate, strong or extreme (Gerberick et al., 2007). Recently, the first amendment of CLP regulation (EC, 2011) allows the allocation of skin sensitisers into subcategory $1 \mathrm{~A}$, strong sensitisers with EC3 value $\leq 2 \%$, or subcategory $1 \mathrm{~B}$ for other skin sensitisers when EC3 value is $>2 \%$. Some threshold limits are provided also for guinea pig methods. This amendment will be applied to substances on December 1, 2012 and to mixtures on June 1, 2015.

\section{Methods}

A detailed analysis was performed on four studies that were submitted to ECHA in corresponding registration dossiers. These studies were selected solely on the basis of their availability to the author, as internal study reports are not public. For reasons of confidentiality, the names of the chemicals are substituted with numbers (Study 1 through 4), as are all references to either the sponsor or the testing facility. The four testing laboratories are different companies and are located in four different EU Member States. For Study no 1 and 2 the original study report was available. Data for Study no 3 and 4 were taken from the IUCLID 5 file and the CSR submitted to ECHA for those substances. The IUCLID 5 file may be incomplete, as the procedure of inserting the data is not GLP regulated and relies upon the discretion of the submitter. The assessment of this small number is not statistically relevant, but provides a first impression of how the LLNA is performed and how well the respective OECD guidelines are followed.

The pilot analysis served to design a strategy for a larger analysis of data that became accessible in the database prepared by ECHA on its website. Until January 2011, about 3400 full registration dossiers were submitted to ECHA. Of these, only about 400 have been included in a publicly accessible database made accessible on the same date. Roughly 100 of these substances belong to the ELINCS (European List of Notified Chemical Substances) database, and about 80 had no official EC number before the pre-registration process began (Rovida and Hartung, 2009). The remaining substances are all part of the EINECS (European Inventory of Existing Commercial chemical Substances) database and are therefore classified as Phase-in substances, according to the REACH definition. It is not clear whether this database is now in its final format, nor is it clear whether the substances made public here are simply the early submissions or if they were selected and how this selection took place. Even though ECHA announced that the database is updated constantly, it does not yet represent the current situation of the chemical market in the European Union. In order to check whether the LLNA was properly applied, however, some of the submitted substances in the database were controlled by analysing sensitive parameters identified in the pilot analysis. This exercise will be repeated when much more data becomes available, i.e. after the next REACH deadline of 1 June 2013. The selection of substances from the database was random. In this dataset of substances, the only possible search query is the chemical name or the CAS/EC number. There is no way to select the chemicals automatically on the basis of a study or of a toxicological result. For this reason, the only possibility was to perform the selection manually, with all the limitations inherent in this process. The four substances that were evaluated in the pilot study were not available in the database, so these are not included in the following selection. It was not possible to verify whether the selected data have been used in read-across for other substances. It is worth noting that the data are published without revision by ECHA, and the information content is identical to that inserted into IUCLID 5 by the submitter. Regarding the results, there is no certainty that these data fully reflect the original study or, more importantly, that the information reported in the original study was transposed fully to the registration dossier (http://apps. echa.europa.eu/registered/registered-sub.aspx). In January 2011, the entire database on the ECHA website was divided into 18 pages, and three or four substances were selected from each page. For each selected substance, the field "7.4.1 skin sensitisation" was analysed. In some cases (12 in total) this field was not present, due perhaps to confidentiality or to other unspecified reasons. When more than one study was submitted per substance, either the study marked as "key study" or the most recent study was considered.

The main purpose of this paper is not to demonstrate whether the LLNA gives a correct prediction, nor is it to judge the reliability of the studies; rather, it is a record of the extent to which testing laboratories follow the guidelines and how they go about it.

\section{Results}

\subsection{Pilot analysis of four LLNA study reports submitted in REACH registration dossiers}

In order to check how the LLNA is applied in ordinary testing laboratories, four reports were analysed in comparison with the OECD guideline. This comparison is detailed in Table 1. 
The first column corresponds to the sections listed in OECD TG 429, while the second column lists the OECD guideline requirements in detail. The reference OECD guideline is the one published in 2002. The latest revision was not taken into consideration, as it was published in July 2010, after all the studies for the 2010 deadline had been performed. The following four columns reflect the four study reports (Study 1 to 4) submitted to ECHA.

Tab. 1: Comparison of four LLNA studies with the official OECD guideline

Details and acronym definitions are in the main text.

\begin{tabular}{|c|c|c|c|c|c|}
\hline & $\begin{array}{l}\text { OECD TG } 429 \text {, } \\
\text { adopted: } \\
\text { April 24, } 2002\end{array}$ & $\begin{array}{l}\text { Study } 1 \\
\text { Data from original } \\
\text { report }\end{array}$ & $\begin{array}{l}\text { Study } 2 \\
\text { Data from original } \\
\text { report }\end{array}$ & $\begin{array}{l}\text { Study } 3 \\
\text { Data extracted } \\
\text { from IUCLID } 5 \\
\text { and CSR }\end{array}$ & $\begin{array}{l}\text { Study } 4 \\
\text { Data extracted } \\
\text { from IUCLID } 5 \\
\text { and CSR }\end{array}$ \\
\hline $\begin{array}{l}\text { Initial } \\
\text { considerations }\end{array}$ & $\begin{array}{l}\text { Applicability Domain: } \\
\text { False negative findings } \\
\text { with certain metals. } \\
\text { False positive findings } \\
\text { with certain skin } \\
\text { irritants. }\end{array}$ & $\begin{array}{l}\text { UVCB sample, i.e. } \\
\text { mixture of unknown } \\
\text { composition. Analytical } \\
\text { characterisation is not } \\
\text { presented claiming } \\
\text { that it was previously } \\
\text { determined by the } \\
\text { sponsor. No further } \\
\text { justification is provided. }\end{array}$ & $\begin{array}{l}\text { Single component, } \\
\text { organic }\end{array}$ & $\begin{array}{l}\text { Single component, } \\
\text { organic }\end{array}$ & $\begin{array}{l}\text { Single component, } \\
\text { inorganic salt }\end{array}$ \\
\hline \multirow[t]{4}{*}{$\begin{array}{l}\text { Description of } \\
\text { the assay }\end{array}$} & $\begin{array}{l}\text { Selection of animal } \\
\text { species: } \\
\text { Young adult female } \\
\text { mice of CBA/Ca or } \\
\text { CBA/J strain. }\end{array}$ & $\begin{array}{l}\text { Female CBA/Ca } \\
\text { mice }\end{array}$ & $\begin{array}{l}\text { Female BALB/c mice; } \\
\text { justification is } \\
\text { provided }\end{array}$ & $\begin{array}{l}\text { Female CBA } \\
\text { mice }\end{array}$ & $\begin{array}{l}\text { Female BALB/c } \\
\text { mice }\end{array}$ \\
\hline & $\begin{array}{l}\text { Age of animals: } \\
\text { At the start of the } \\
\text { study animals should } \\
\text { be between } \\
8-12 \text { weeks old. }\end{array}$ & $7-8$ weeks old & $8-10$ weeks old & Not reported & 6-8 weeks old \\
\hline & $\begin{array}{l}\text { Weight of animals: } \\
\text { Weight variation of } \\
\text { the animals should } \\
\text { not exceed } 20 \% \text { of } \\
\text { the mean weight. }\end{array}$ & $\begin{array}{l}\text { Initial weight: } \\
15.1 \mathrm{~g}-18.8 \mathrm{~g} ; \\
\text { statistically significant } \\
\text { increase in the weight } \\
\text { of all animals during } \\
\text { the study, however } \\
\text { always within the } \\
20 \% \text { variation. }\end{array}$ & $\begin{array}{l}\text { Initial weight: } \\
15 \mathrm{~g}-25 \mathrm{~g} \text {; } \\
\text { controlled at the } \\
\text { beginning and at } \\
\text { the end of the study. }\end{array}$ & Not reported & Not reported \\
\hline & $\begin{array}{l}\text { Acceptable deviations: } \\
\text { Other strains and } \\
\text { males when sufficient } \\
\text { data are generated to } \\
\text { demonstrate that } \\
\text { significant differences } \\
\text { do not exist. }\end{array}$ & None reported & None reported & None reported & $\begin{array}{l}\text { Non-radioactive } \\
\text { procedure, as } \\
\text { described now in } \\
\text { OECD TG 442b. } \\
\text { No mention about } \\
\text { different mouse strain } \\
\text { and different age. }\end{array}$ \\
\hline \multirow[t]{3}{*}{$\begin{array}{l}\text { Housing and } \\
\text { feeding } \\
\text { conditions }\end{array}$} & $\begin{array}{l}\text { Animals should be } \\
\text { individually housed. }\end{array}$ & $\begin{array}{l}\text { Animals are housed } \\
\text { in groups } \\
\text { of } 2-3 \text { in cages. } \\
\text { Detailed description of } \\
\text { the cage is provided. }\end{array}$ & $\begin{array}{l}\text { Animals are housed } \\
\text { in groups of } \\
\text { maximum } 6 \text { in cages. }\end{array}$ & Individually housed & Not reported \\
\hline & $\begin{array}{l}\text { Temperature: } \\
22^{\circ} \mathrm{C}\left( \pm 3^{\circ} \mathrm{C}\right)\end{array}$ & $\begin{array}{l}20-21^{\circ} \mathrm{C} \text { (monitored } \\
\text { every } 15 \text { minutes) }\end{array}$ & $22^{\circ} \mathrm{C}\left( \pm 3^{\circ} \mathrm{C}\right)$ & $20-24^{\circ} \mathrm{C}$ & Not reported \\
\hline & $\begin{array}{l}\text { Humidity: } \\
50-60 \% \text { (never less } \\
\text { than } 30 \% \text { or } \\
\text { more than } 70 \% \text { ) }\end{array}$ & $\begin{array}{l}42-64 \% \text { (monitored } \\
\text { every } 15 \text { minutes) }\end{array}$ & $30-70 \%$ & $18-65 \%$ & Not reported \\
\hline
\end{tabular}




\begin{tabular}{|c|c|c|c|c|c|}
\hline & $\begin{array}{l}\text { OECD TG } 429 \text {, } \\
\text { adopted: } \\
\text { April 24, } 2002\end{array}$ & $\begin{array}{l}\text { Study } 1 \\
\text { Data from original } \\
\text { report }\end{array}$ & $\begin{array}{l}\text { Study } 2 \\
\text { Data from original } \\
\text { report }\end{array}$ & $\begin{array}{l}\text { Study } 3 \\
\text { Data extracted } \\
\text { from IUCLID } 5 \\
\text { and CSR }\end{array}$ & $\begin{array}{l}\text { Study } 4 \\
\text { Data extracted } \\
\text { from IUCLID } 5 \\
\text { and CSR }\end{array}$ \\
\hline \multirow[t]{2}{*}{$\begin{array}{l}\text { Housing and } \\
\text { feeding } \\
\text { conditions }\end{array}$} & $\begin{array}{l}\text { Artificial lighting: } \\
12 \text { hours light/ } \\
12 \text { hours dark }\end{array}$ & $\begin{array}{l}12 \text { hours light/ } \\
12 \text { hours dark }\end{array}$ & $\begin{array}{l}12 \text { hours light/ } \\
12 \text { hours dark }\end{array}$ & $\begin{array}{l}12 \text { hours light/ } \\
12 \text { hours dark }\end{array}$ & Not reported \\
\hline & $\begin{array}{l}\text { Feeding with } \\
\text { conventional lab diet } \\
\text { and unlimited supply } \\
\text { of drinking water }\end{array}$ & $\begin{array}{l}\text { Ad libitum throughout } \\
\text { the study }\end{array}$ & $\begin{array}{l}\text { Ad libitum throughout } \\
\text { the study }\end{array}$ & $\begin{array}{l}\text { Ad libitum throughout } \\
\text { the study }\end{array}$ & Not reported \\
\hline \multirow[t]{3}{*}{$\begin{array}{l}\text { Preparation of } \\
\text { animals }\end{array}$} & $\begin{array}{l}\text { Random selection of } \\
\text { the animal, marked } \\
\text { but not on ears }\end{array}$ & $\begin{array}{l}\text { Selection upon } \\
\text { arrival; identified by } \\
\text { subcutaneous implant } \\
\text { of a microchip }\end{array}$ & $\begin{array}{l}\text { Random selection; } \\
\text { felt tip marking }\end{array}$ & $\begin{array}{l}\text { Random selection; } \\
\text { no details about } \\
\text { identification tools }\end{array}$ & Not reported \\
\hline & $\begin{array}{l}\text { Acclimatisation: } \\
5 \text { days before starting } \\
\text { the treatment }\end{array}$ & 8 days as minimum & Not reported & $\begin{array}{l}5 \text { days before starting } \\
\text { the treatment }\end{array}$ & Not reported \\
\hline & $\begin{array}{l}\text { Prior to the start of } \\
\text { the experiments, all } \\
\text { animals are examined } \\
\text { to ensure that they } \\
\text { have no observable } \\
\text { skin lesions. }\end{array}$ & $\begin{array}{l}\text { All animals are } \\
\text { checked for viability. } \\
\text { No mention about } \\
\text { skin lesions. }\end{array}$ & Not reported & Not reported & Not reported \\
\hline \multirow[t]{3}{*}{ Reliability Check } & $\begin{array}{l}\text { Positive control } \\
\text { response should be } \\
\text { clear but not } \\
\text { excessive. Preferred } \\
\text { substances: hexyl } \\
\text { cinnamic aldehyde } \\
\text { and mercapto- } \\
\text { benzothiazole }\end{array}$ & $\begin{array}{l}\text { Positive control: } \\
\text { hexyl cinnamic } \\
\text { aldehyde }\end{array}$ & $\begin{array}{l}\text { Dinitrochlorobenzene; } \\
\text { final measured } \\
\text { SI= } 12 \text { at } 0.5 \% \mathrm{w} / \mathrm{v}\end{array}$ & $\begin{array}{l}\text { Positive control: } \\
\text { hexyl cinnamic } \\
\text { aldehyde }\end{array}$ & Dinitrochlorobenzene \\
\hline & $\begin{array}{l}\text { Positive controls not } \\
\text { necessary when } \\
\text { historic data are } \\
\text { available. }\end{array}$ & $\begin{array}{l}\text { Periodically checked. } \\
\text { The latest was } \\
5 \text { months before the } \\
\text { beginning of the study. }\end{array}$ & With the test & With the test & With the test \\
\hline & $\begin{array}{l}\text { Recommended vehicle } \\
\text { for positive control: } \\
\text { acetone:olive oil (4:1). } \\
\text { Others may be } \\
\text { accepted in special } \\
\text { circumstances. }\end{array}$ & $\mathrm{AOO}$ & $\begin{array}{l}\text { DAE } 433 \\
\text { ( } 40 \% \text { dimethyla- } \\
\text { cetamide, } \\
30 \% \text { acetone and } \\
30 \% \text { ethanol). } \\
\text { No justification is } \\
\text { provided. }\end{array}$ & $\mathrm{AOO}$ & $\mathrm{AOO}$ \\
\hline \multirow[t]{2}{*}{$\begin{array}{l}\text { Number of } \\
\text { animals and } \\
\text { dose levels }\end{array}$} & $\begin{array}{l}\text { Animals per } \\
\text { dose group: } \\
\text { minimum of } 4 \\
\text { (minimum of } 5 \text { when } \\
\text { individual endpoints } \\
\text { are taken) }\end{array}$ & 5 animals per group & $\begin{array}{l}5 \text { animals per group } \\
\text { plus } 2 \text { as reserve }\end{array}$ & 5 animals per group & 4 animals per group \\
\hline & $\begin{array}{l}\text { Minimum of three } \\
\text { concentrations of the } \\
\text { test substance plus } \\
\text { negative control }\end{array}$ & $\begin{array}{l}0 \%, 25 \%, 50 \%, \\
100 \%\end{array}$ & $\begin{array}{l}0 \%, 0.3 \%, 3 \%, 30 \% \\
\text { General details about } \\
\text { dose selection } \\
\text { (30\% concentration } \\
\text { was tested in a } \\
\text { preliminary test with } \\
\text { no evident sign of } \\
\text { systemic toxicity). }\end{array}$ & $\begin{array}{l}0 \%, 0.5 \%, 10 \%, 25 \% \\
\text { No justification for } \\
\text { this selection } \\
\text { of concentrations } \\
\text { is provided. }\end{array}$ & $\begin{array}{l}0 \%, 1 \%, 3 \%, 10 \% \\
\text { No justification for } \\
\text { this selection } \\
\text { of concentrations } \\
\text { is provided. }\end{array}$ \\
\hline
\end{tabular}




\begin{tabular}{|c|c|c|c|c|c|}
\hline & $\begin{array}{l}\text { OECD TG } 429 \text {, } \\
\text { adopted: } \\
\text { April 24, } 2002\end{array}$ & $\begin{array}{l}\text { Study } 1 \\
\text { Data from original } \\
\text { report }\end{array}$ & $\begin{array}{l}\text { Study } 2 \\
\text { Data from original } \\
\text { report }\end{array}$ & $\begin{array}{l}\text { Study } 3 \\
\text { Data extracted } \\
\text { from IUCLID } 5 \\
\text { and CSR }\end{array}$ & $\begin{array}{l}\text { Study } 4 \\
\text { Data extracted } \\
\text { from IUCLID } 5 \\
\text { and CSR }\end{array}$ \\
\hline \multirow[t]{2}{*}{$\begin{array}{l}\text { Number of } \\
\text { animals and } \\
\text { dose levels }\end{array}$} & $\begin{array}{l}\text { Concentrations: } \\
\text { highest concentration } \\
\text { without systemic } \\
\text { toxicity and excessive } \\
\text { local skin irritation }\end{array}$ & $\begin{array}{l}\text { Preliminary test with } \\
2 \text { animals treated } \\
\text { at } 100 \% \text { of sample }\end{array}$ & $\begin{array}{l}\text { Preliminary test with } \\
3 \text { animals at } 30 \% \text { con- } \\
\text { centration of test item }\end{array}$ & $\begin{array}{l}\text { Systemic toxicity } \\
\text { evaluated directly } \\
\text { during the experiment }\end{array}$ & Not reported \\
\hline & $\begin{array}{l}\text { Vehicle, order of } \\
\text { preference: } \\
\text { AOO, dimethyl- } \\
\text { formamide, methyl } \\
\text { ethyl ketone, } \\
\text { propylene glycol and } \\
\text { dimethyl sulphoxide. } \\
\text { Avoid } 100 \% \text { water. }\end{array}$ & $\mathrm{AOO}$ & $\begin{array}{l}\text { DAE } 433 \text {, mixture of } \\
40 \% \text { dimethylace- } \\
\text { tamide, } 30 \% \text { acetone } \\
\text { and } 30 \% \text { ethanol; } \\
\text { no justification } \\
\text { is provided. }\end{array}$ & $\begin{array}{l}\text { Tetrahydrofuran; } \\
\text { different from } \\
\text { positive control. } \\
\text { No justification of } \\
\text { this selection. }\end{array}$ & $\begin{array}{l}\text { Petroleum ether and } \\
\text { olive oil }(4: 1) ; \\
\text { different from } \\
\text { positive control. } \\
\text { No justification of } \\
\text { this selection. } \\
\text { Some concerns exist } \\
\text { about substance } \\
\text { solubility. }\end{array}$ \\
\hline \multirow[t]{7}{*}{$\begin{array}{l}\text { Experimental } \\
\text { schedule }\end{array}$} & $\begin{array}{l}\text { Day } 1 \text { : Individually } \\
\text { identify and record the } \\
\text { weight of each animal. } \\
\text { Open application of } \\
25 \mu \text { of the appropriate } \\
\text { dilution of the test } \\
\text { substance, the vehicle } \\
\text { alone, or the positive } \\
\text { control (as appro- } \\
\text { priate), to the dorsum } \\
\text { of each ear. }\end{array}$ & Same procedure & Same procedure & Same procedure & Same procedure \\
\hline & $\begin{array}{l}\text { Days } 2 \text { and } 3 \text { : } \\
\text { Repeat the application } \\
\text { procedure carried } \\
\text { out on day } 1 .\end{array}$ & Same procedure & Same procedure & Same procedure & Same procedure \\
\hline & $\begin{array}{l}\text { Days } 4 \text { and } 5: \\
\text { No treatment }\end{array}$ & Same procedure & Same procedure & Same procedure & $\begin{array}{l}\text { According to OECD } \\
\text { TG } 442 b \text { (no treatment } \\
\text { only on Day 4) }\end{array}$ \\
\hline & $\begin{array}{l}\text { End of test (Day 6): } \\
\text { Weigh each animal. }\end{array}$ & Done & Done & Not reported & Not reported \\
\hline & $\begin{array}{l}\text { Inject T-thymidine } \\
\text { via tail vein. }\end{array}$ & Same procedure & Same procedure & Same procedure & $\begin{array}{l}\text { According to } \\
\text { OECD TG 442b } \\
\text { (Injection of 5-bromo- } \\
\text { 2-deoxyuridine (BrdU) } \\
\text { intraperitoneally) }\end{array}$ \\
\hline & $\begin{array}{l}\text { Kill the animal } \\
5 \text { hours later. }\end{array}$ & Same procedure & Same procedure & Same procedure & $\begin{array}{l}\text { According to } \\
\text { OECD TG } 442 b \\
\text { (Sacrifice } 24 \text { hours } \\
\text { after BrdU injection) }\end{array}$ \\
\hline & $\begin{array}{l}\text { Draining auricular } \\
\text { lymph nodes are } \\
\text { excised and pooled. }\end{array}$ & $\begin{array}{l}\text { Same procedure; } \\
\text { single animal } \\
\text { measured. }\end{array}$ & $\begin{array}{l}\text { Same procedure; } \\
\text { single animal } \\
\text { measured. }\end{array}$ & $\begin{array}{l}\text { Same procedure; } \\
\text { single animal } \\
\text { measured. }\end{array}$ & $\begin{array}{l}\text { Same procedure; } \\
\text { single animal } \\
\text { measured. }\end{array}$ \\
\hline \multirow[t]{2}{*}{$\begin{array}{l}\text { Preparation of } \\
\text { cell suspension }\end{array}$} & $\begin{array}{l}\text { Lymph node cells } \\
\text { are washed } \\
\text { twice with PBS. }\end{array}$ & Same procedure & Same procedure & Same procedure & $\begin{array}{l}\text { According to } \\
\text { OECD TG } 442 b \\
\text { (preparation of a single } \\
\text { cell suspension of } \\
\text { lymph node cells) }\end{array}$ \\
\hline & Precipitated with TCA & Same procedure & Same procedure & Same procedure & $\begin{array}{l}\text { According to } \\
\text { OECD TG 442b } \\
\text { (not required) }\end{array}$ \\
\hline
\end{tabular}




\begin{tabular}{|c|c|c|c|c|c|}
\hline & $\begin{array}{l}\text { OECD TG } 429 \text {, } \\
\text { adopted: } \\
\text { April 24, } 2002\end{array}$ & $\begin{array}{l}\text { Study } 1 \\
\text { Data from original } \\
\text { report }\end{array}$ & $\begin{array}{l}\text { Study } 2 \\
\text { Data from original } \\
\text { report }\end{array}$ & $\begin{array}{l}\text { Study } 3 \\
\text { Data extracted } \\
\text { from IUCLID } 5 \\
\text { and CSR }\end{array}$ & $\begin{array}{l}\text { Study } 4 \\
\text { Data extracted } \\
\text { from IUCLID } 5 \\
\text { and CSR }\end{array}$ \\
\hline $\begin{array}{l}\text { Preparation of } \\
\text { cell suspension }\end{array}$ & Measure DPM & Same procedure & Same procedure & Same procedure & $\begin{array}{l}\text { According to } \\
\text { OECD TG } 442 b \\
\text { (use of a commercial } \\
\text { ELISA kit) }\end{array}$ \\
\hline \multirow[t]{3}{*}{$\begin{array}{l}\text { Calculation of } \\
\text { results, i.e. } \\
\text { Stimulation } \\
\text { Index (SI) }\end{array}$} & $\begin{array}{l}\text { Pooled approach: } \\
\text { Pooled radioactive } \\
\text { incorporation of each } \\
\text { treatment group } \\
\text { divided by the pooled } \\
\text { radioactive incor- } \\
\text { poration of vehicle } \\
\text { control group. }\end{array}$ & Not applied & Not applied & Not applied & Not applied \\
\hline & $\begin{array}{l}\text { Individual approach: } \\
\text { Mean of radioactive } \\
\text { incorporation of each } \\
\text { animal in a group } \\
\text { divided by the mean } \\
\text { of radioactive } \\
\text { incorporation of each } \\
\text { animal in the vehicle } \\
\text { control group. }\end{array}$ & Same procedure & Same procedure & Same procedure & $\begin{array}{l}\text { According to } \\
\text { OECD TG 442b (mean } \\
\text { BrdU labelling index/ } \\
\text { mouse divided by the } \\
\text { mean BrdU labelling } \\
\text { index/mouse in the } \\
\text { vehicle control group) }\end{array}$ \\
\hline & $\begin{array}{l}\text { SI is derived by } \\
\text { dividing the mean } \\
\text { DPM/mouse within } \\
\text { each test substance } \\
\text { group by the mean } \\
\text { DPM/mouse for the } \\
\text { vehicle control group. }\end{array}$ & Same procedure & Same procedure & Same procedure & Same procedure \\
\hline $\begin{array}{l}\text { Data and } \\
\text { reporting }\end{array}$ & Detailed & $\begin{array}{l}\text { Detailed enough, } \\
\text { even though some } \\
\text { deviations are not } \\
\text { justified. } \\
\text { Characterisation of } \\
\text { the test sample upon } \\
\text { the responsibility } \\
\text { of the sponsor. }\end{array}$ & $\begin{array}{l}\text { Detailed enough, } \\
\text { even though } \\
\text { important deviations } \\
\text { are not justified. }\end{array}$ & $\begin{array}{l}\text { Data retrieved } \\
\text { from IUCLID } 5 . \\
\text { Much information } \\
\text { is missing. }\end{array}$ & $\begin{array}{l}\text { Data retrieved } \\
\text { from IUCLID } 5 . \\
\text { Much information } \\
\text { is missing. }\end{array}$ \\
\hline $\begin{array}{l}\text { Total number } \\
\text { of animals used }\end{array}$ & $\begin{array}{l}\text { Required: } \\
\text { Minimum } 16 \\
\text { ( } 3 \text { concentrations, } \\
\text { negative control, } \\
4 \text { animals per group) } \\
\text { Maximum } 25 \\
\text { ( } 3 \text { concentrations, } \\
\text { negative and positive } \\
\text { controls, } 5 \text { animals } \\
\text { per group) }\end{array}$ & $\begin{array}{l}22 \text { mice } \\
5 \text { animals per group } \\
\text { per } 3 \text { testing con- } \\
\text { centrations plus } \\
\text { negative control plus } \\
\text { two mice for the } \\
\text { pre-screen test }\end{array}$ & $\begin{array}{l}30 \text { mice } \\
5 \text { animals per group } \\
\text { per } 3 \text { testing con- } \\
\text { centrations plus } \\
\text { negative and positive } \\
\text { controls plus three } \\
\text { mice for the pre- } \\
\text { screen test and two } \\
\text { as reserve }\end{array}$ & $\begin{array}{l}25 \text { mice } \\
5 \text { animals per group } \\
\text { per } 3 \text { testing con- } \\
\text { centrations plus } \\
\text { negative and positive } \\
\text { controls. } \\
\text { No information about } \\
\text { pre-screen test }\end{array}$ & $\begin{array}{l}20 \text { mice } \\
\text { No information about } \\
\text { pre-screen test }\end{array}$ \\
\hline Result & & Negative & Positive & Negative & Positive \\
\hline
\end{tabular}

In each row the OECD requirement is followed by the correspondent data present in the four studies. Deviations from OECD guidelines are marked in bold on a grey background. It should be noted that all four studies were performed by certified laboratories. The term "fully GLP compliant study" means that the testing laboratory fully applies a protocol that has been pre- viously agreed upon with the sponsor. This signed protocol may differ from the OECD guideline, so a lack of comments about how it differs from the guideline does not necessarily mean it is a noncompliant procedure, as it follows the signed protocol. However, this is not fully true if compliance with the OECD guideline is mentioned in the protocol, as is the case for both 
Studies no 1 and 2. No such information is available for Studies 3 and 4. However, it is supposed that those new tests are performed for REACH purposes and thus a good testing facility should prepare a study protocol according to the specific needs. In fact, both in IUCLID 5 windows and in the ECHA guidelines there is a specific requirement to indicate any deviations from the official guideline, in this case OECD TG 429. Study 4 differs in that it follows a method that is a non-radioactive variation of the original LLNA method, i.e. the BrdU-ELISA method (Basketter et al., 2008). This protocol introduces only minor changes to the standard LLNA protocol and is already well accepted in the scientific world. Now, the OECD TG 442b fully endorses its use. OECD TG 442b was used in Table 1 as the reference method for Study 4.

\section{Application domain}

Initial considerations, and maybe the most important, concern limitations in the applicability domain of LLNA. The LLNA validation report (NTP, 1999) highlights some false negative results obtained with certain metals and some false positive results obtained with strong irritants. The reason why some metals interfere with the test is still a matter of discussion, even though a recent retrospective analysis of LLNA performance indicates that the problem is restricted to nickel salts (ICCVAM, 2009). On the other hand, it is generally accepted that strong irritants may elicit an immunological reaction that can be confused with a sensitisation property (NTP, 1999). This issue is often addressed in a preliminary dose-ranging study to evaluate the highest possible chemical concentration that causes no systemic toxicity or irritation. Another consideration with regard to the chemical nature of the tested substance is that impurities may trigger an allergic reaction. Precise analytical characterisation of the test sample is thus of utmost importance.

In the REACH context, the definition of the type of chemical is always well categorised in the registration dossier. In fact, in IUCLID 5, the identification page of a substance asks for information on the type of substance. This is split between composition and origin, with a selection between:

1. Composition

- Mono-constituent substance

- Multi-constituent substance

- UVCB (Unknown or Variable composition, Complex reaction products or Biological materials)

- Polymer

2. Origin

- Element

- Inorganic

- Organic

- Organometallic

- Petroleum product

Mono-constituent substances must have a chemical purity higher than $80 \%$; otherwise they are either multiconstituent or UVCB. The second form of classification relates to whether the substance is organic or inorganic. In the reports analysed in Table 1, no explicit statements are made on possible limitations given by the type of tested substance, even though one of the four substances is an inorganic salt and another is a UVCB The OECD TG 429 only explicitly excludes mixtures from the applicability domain, and the list of chemicals that were analysed for the validation of LLNA does include UVCB samples. The real problem is that UVCB substances may have a broad composition range and something should be said to justify the extrapolation of the result of a test performed on a specific sample to the entire UVCB substance composition covered by the registration dossier. Typically, UVCBs may be a reaction product where 3,4 or even more components may be present in a range between 0-40\%. From a biochemical point of view, it is very different if the tested sample has either 0 or $40 \%$ of a chemical. The result may be correct, but at least some further reasoning should be given before simply accepting the result as it is for the whole composition range covered by the UVCB definition. Hopefully this issue is addressed somewhere else in the CSR of that substance.

A more serious deviation was observed in Study 4, which was performed on an inorganic salt, i.e. containing metal ions. In the report, nothing is said to justify the choice.

\section{Animal selection and housing}

The next section of Table 1 looks at the description of the assay and general considerations about the animals, with the first row addressing animal species. The CBA mouse strain was selected by OECD as the preferred choice, since a systematic comparison of data to other strains was missing. Two of the four studies apply the correct strain. The other two studies use another mouse strain (Balb/c instead of CBA), which also conflicts with the indications of OECD 442b. The different choice could be justified, as the BALB/c is the strain commonly used in NTP studies (NTP, 2005), and subsequent studies demonstrated the equivalence of the two species for the LLNA (Woolhiser et al., 2000; Burns et al., 2010). Some concerns remain, however, as pointed out in the validation report (NTP, 1999) and in the ECHA guideline (ECHA, 2008). The validation reports of both the standard LLNA and the BrdU-ELISA test methods state explicitly that the CBA strain is the only accepted strain for new studies. No justification is provided for the different selection in Study 4, generating a second important deviation from the acceptability criteria. On the other hand, Study 2 justifies the selection of the BALB/c strain on account of the availability of historical internal control data.

With regard to age of the animals, OECD asks for animals aged 8-12 weeks. Study 2 is compliant, Study 3 does not report the information, and the other two studies are out of the range. The LLNA protocol that was submitted to ICCVAM for validation in 1997 accepted 7-12 week old mice. Even considering this slightly broader range, Study 4 is still out. Both studies should provide justification for this choice.

The weight of the animals is generally recorded in any in vivo experiment, and it can be assumed that this also was done in cases where no data are reported. Reporting initial weight of the mice is requested in the IUCLID 5 file and is confirmed in the ECHA guideline on compiling a robust study summary (ECHA Practical Guide 3), so those two studies are still negligent. 
In 3 out of the 4 studies, animals are grouped in cages instead of being individually housed. This can easily be disregarded as a non-compliant procedure, however, as the revision of the OECD guideline 429 (July 2010) now permits this possibility.

The reporting of other physical parameters, such as temperature, light cycle, etc. seems almost overly compliant. Only one data point is out of range, and that is the humidity in Studies 2 and 3. A very low humidity value may affect the absorption rate, and this deserves greater consideration. It may be hopefully assumed that this value is not the result of specific measures but rather a broad range that includes normal lab conditions. However, it is again another inaccurate parameter.

\section{Choice of positive control and vehicle}

As a general rule, any test should run a positive control to demonstrate that the experiment is performed well and personnel are operating correctly. For ethical reasons, in vivo experiments should have a positive control only when absolutely necessary, and that is why protocols may accept historical data to reduce the number of animals and to minimise the distress that is always correlated with a positive outcome. It seems that only the protocol of Study 1 adopted this procedure. Even worse, two of the four studies used dinitrochlorobenzene (DNCB) as a positive control. This is not acceptable for two reasons: the OECD guideline recommends the use of either hexylcinnamic aldehyde (HCA) or mercaptobenzothiazole and, as an alternative, a different positive control for which the response is not excessive. DNCB does not suit the purpose, as it is an extreme sensitiser, while HCA is only a weak sensitiser. The use of such a strong sensitiser is not appropriate to demonstrate that the procedure is correctly performed, and it subjects animals to unnecessary distress. OECD TG 442b recommends the use of either HCA or eugenol instead of mercaptobenzothiazole and does not include DNCB for the same reasons.

All protocols but one correctly dissolve the positive controls in an acetone/olive oil (4:1 v/v) (AOO) vehicle, but only Study 1 applies the rule that the vehicle used for the positive control should also be used to dissolve the tested substance. The OECD guideline suggests the use of AOO as a first choice, followed by other possibilities which may be required to fully dissolve the tested sample. The order of preference is AOO, dimethylformamide (DMF), methyl ethyl ketone, propylene glycol, dimethyl sulphoxide. Wholly aqueous vehicles are to be avoided. These solvents should be able to dissolve most chemicals. The vehicle may strongly affect the final result (Basketter et al., 2001, Jowsey et al., 2008), and therefore this is regarded as a very sensitive parameter. As usual, the use of another vehicle may be accepted when valid scientific justification can be provided. Three reports do not use the same solvents for the tested substances and the positive controls with no justification. The vehicle alone is always used as negative control, but this is not enough to justify the selection. Further, Study 4, which also applies different solvents for tested substance and positive control, applies petroleum ether and olive oil (4:1) as solvent for the inorganic salt, which probably re- sults in a suspension rather than a solution. This is unsuitable application of the substance to mouse skin.

\section{Test concentration}

The concentration of substance to be applied to the mice should be selected starting from the highest concentration that induced no sign of systemic toxicity or irritation. Study 1 apparently set no limitations as the substance is tested at $100 \%$ as the highest level. Like Study 1, also Study 2 reports performing a pre-screen test, which leads to a selection of $30 \%$ as the highest dose level. Nothing is specified in Studies 3 and 4. Even though not reported here, it is known that the substance tested in Study 4 is toxic, and this may justify the selection of $10 \%$ as the highest dose. Both the revised version of OECD TG 429 and OECD TG 442b describe clearly how to perform the pre-screen test.

\section{Information content}

Data and reporting are partially satisfactory only in Studies 1 and 2: still some deviations from the guideline are not fully justified. Studies 3 and 4 were retrieved directly from the IUCLID 5 file, and no conclusions can be drawn about the structure of the final reports. However, this still constitutes an omission, because ECHA requires that all relevant data be fully transposed into IUCLID 5. It also should be noted that IUCLID 5 refers to the specific guideline (i.e. in this case OECD TG 429 and TG 442b) and not to the protocol that backs the experiment. The GLP compliance statement, although mandatory, is not sufficient: GLP means that an experiment follows a protocol that was signed by both the sponsor and the test facility. If this protocol deviates from the relevant OECD guideline, the study will still be GLP compliant, but it will not be suitable for REACH purposes. Too often the GLP compliance statement is considered synonymous with a fully reliable study, but this is not true. GLP statements are necessary, but they must be coupled with a suitable study design which needs to be focused on the purpose of the test, i.e. be compliant with the respective guideline if the data is to be used for REACH.

\section{Animal number}

Last but not least, the total number of animals used per experiment warrants some consideration. The number of animals per group is five except in Study 4, where it is not provided. The choice of five animals with individual measurement of the lymph nodes is the only procedure accepted by the Environmental Protection Agency (EPA, 2003), but four mice per group would be sufficient for the OECD guideline. The decision to use more animals is acceptable only if the same study will be submitted to the US. Therefore, the use of 5 animals per group may be justified as a preventive measure, even if the awareness of this decision is questionable, as no clear statements are made on this. Study number 2 mentions the use of two more animals that are included in the study as reserve. However, the sense of having reserve animals, which are sacrificed at the end of the experiment although they were not used, is not compliant with the aim to use as few animal as possible. 


\section{Interpretation}

The final result is negative for studies 1 and 3 and positive for studies 2 and 4, leading to classification of the substances as H317, may cause an allergic skin reaction, and labeling with the exclamation mark pictogram. Conclusions as to the reliability of these results, considering all the deviations from the guidelines, is beyond the scope of this paper, which aims only to monitor how labs are conducting experiments. The only comment to be made concerns Study 4, which is positive even though the test substance was an inorganic salt for which the LLNA is suspected of returning false negative results. This result makes the study acceptable in spite of the concern. However, it is interesting to note that the correspondent oxide was also tested using the LLNA (data not reported here), with negative results. For this reason, the correspondent oxide was not classified as a skin sensitiser (H317). Therefore, either a sensitisation reaction is triggered only by the metal in its ionic form or the oxide is not absorbed through the skin. Even though little information is known, as the full CSR is not available, a simple proposal for a more effective testing strategy would be the following (Fig. 1). The first step of the tier approach could have been to evaluate whether the oxide can penetrate the skin barrier with a suitable skin absorption study. Skin absorption is determined by OECD TG 428 that also is implemented in Regulation 440/2008. As the salt is classified as a strong sensitiser, it can be assumed that the oxide also will be a sensitiser if absorbed at a rate higher than $0.1 \%$ in 24 hours. If absorption is negligible, the substance can be safely regarded as a non sensitiser. In this way, the real hazard of the substance would have been understood, and the LLNA test could have been waived, saving both money and mice. The complete set of data is not available, and therefore the above reasoning may be misleading. It is evident, however, that this route was not considered, confirming a sense that the actors involved in the REACH process give minimal consideration to non animal alternatives (Rovida, 2010) and evidence no interest in the real toxicological mechanisms of substances. Incidentally, this metal oxide is classified as a respiratory sensitiser (H334), as a result of epidemiological evidence from exposed workers!

It is evident that a change in mindset is often required. A more rigorous approach to the toxicological assessment of chemicals - an approach that no longer depends on a single method that gives a yes/no answer - is one of the goals of evidence-based toxicology (Hoffman and Hartung, 2006). This new toxicology turns to performance characteristics, mechanistic understanding, and extended quality assurance to provide a solid foundation for any decision about the possible hazards related to the use of a chemical substance.

\subsection{Analysis of the ECHA database}

75 of 400 substances were randomly selected from the ECHA database. The content found in section 7.4.1 is presented in Table 2, which lists the types of methods applied to assess skin sensitisation. All of the entries classified as "other" were different types of publications and none of them were based on new in vitro tests or in silico analysis. In 12 cases, the field 7.4.1 was not present for unknown reasons. For 20 of the 75 selected substances, the indicated test guideline was the OECD 429. Few of these studies were based on published data; most were derived from company study reports..

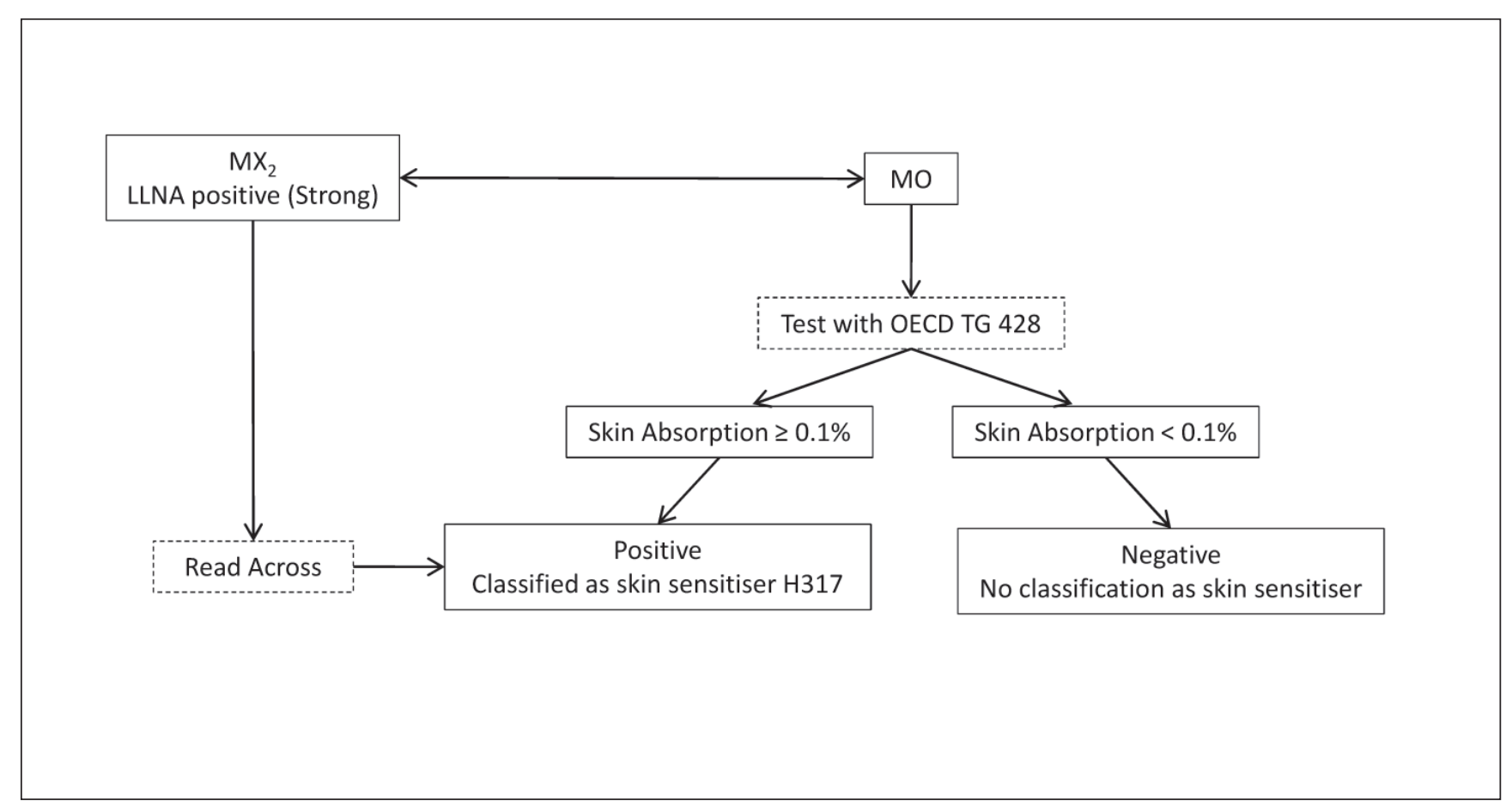

Fig. 1: Possible testing strategy for skin sensitization

$\mathrm{MX}$ : metal salt; $\mathrm{MO}$ : metal oxide 
Tab. 2: Distribution of methods for the evaluation of skin sensitisation (paragraph 7.4.1 in the database)

\begin{tabular}{|l|l|l|l|l|l|}
\hline & $\begin{array}{l}\text { OECD TG 429 } \\
\text { (LLNA) }\end{array}$ & OECD TG 406 & $\begin{array}{l}\text { Other (human data, } \\
\text { read across, etc.) }\end{array}$ & None & Total assessed \\
\hline Number of studies & 20 & 31 & 12 & 12 & 75 \\
\hline Relative $\%$ & $27 \%$ & $41 \%$ & $16 \%$ & $16 \%$ & $100 \%$ \\
\hline
\end{tabular}

All records that had the LLNA marked as an experimental study for skin sensitisation were investigated further. Table 3 lists the time frame in which the LLNA was performed. As only one was done in 2006, two in 2007, and all others in a period following 2008, it can be assumed that the LLNAs were done primarily for REACH purposes.

Tab. 3: Distribution of report dates for LLNA selection

\begin{tabular}{|l|l|}
\hline Year & Number of studies \\
\hline 2006 & 1 \\
\hline 2007 & 2 \\
\hline$\geq 2008$ & 17 \\
\hline
\end{tabular}

Whenever the LLNA was applied, the fields applicability domain, positive control selection, choice of vehicle and number of mice used were considered. Other non-conformities found in the four previous reports were not analysed here, as they were considered of lesser interest and the correspondent data often were not reported in the database (humidity, age of mice, etc.).

\section{Applicability domain}

With regard to the applicability domain (Tab. 4), ten of the reports were on mono-constituent organic substances, seven were UVCB and the rest were organometallic or inorganic. The test guideline does not explicitly exclude UVCB, mentioning only that mixtures are not covered. However, validation of the LLNA for mixtures is ongoing (ICCVAM, 2009) and, therefore, data acquired on UVCB samples can be fully acceptable, even if no justification about how to accept the result of a specific sample as representative for the whole UVCB composition range is presented in the final report. The organometallic substance and the two inorganic salts are of greater concern. One of the inorganic salts gave negative results, so the question remains as to whether this was a true or a false negative. This question was

Tab. 4: Distribution of substance types for LLNA selection

\begin{tabular}{|l|l|}
\hline Category of the tested chemical & Number of studies \\
\hline UVCB & 7 \\
\hline Organic, mono-component & 10 \\
\hline Organometallic & 1 \\
\hline Inorganic, mono component & 2 \\
\hline
\end{tabular}

not addressed, and the LLNA result is directly taken to justify the "non sensitiser" classification.

\section{Positive control selection}

Selection of positive controls is reported in Table 5. All but two of the studies included a positive control. Apparently, the positive control was always done simultaneously with the experiment. Only in one case, it is mentioned that the testing facility found many positive substances, so it is assumed that no additional positive control was done in that case. Regarding the positive control, the good news is that the majority of the laboratories correctly adopted HCA, with only one using DNCB. The sensitisation category of the positive controls in Table 5 was taken from the ICCVAM validation report (NTP, 1999). Phenylacetaldehyde is quite uncommon, but as it is a moderate skin sensitiser with an EC3 value of 3\%, it can be assumed to be acceptable, even though nothing was mentioned in the field "deviations from the guideline." The other recommended choice in the guideline is mercaptobenzothiazole, a moderate sensitiser with an EC3 value of 7\%, which was never applied.

\section{Tab. 5: Distribution of positive control selection}

\begin{tabular}{|l|l|l|}
\hline Positive controls & $\begin{array}{l}\text { Number } \\
\text { of studies }\end{array}$ & $\begin{array}{l}\text { Sensitisation } \\
\text { Category }\end{array}$ \\
\hline DNCB & 1 & Extreme \\
\hline HCA & 15 & Weak \\
\hline Phenylacetaldehyde & 1 & Moderate \\
\hline $\begin{array}{l}\text { List of many positive chemicals } \\
\text { tested in the lab }\end{array}$ & 1 & - \\
\hline Not reported & 2 & - \\
\hline
\end{tabular}

\section{Choice of vehicle}

Analysis of the selection of vehicle (Tab. 6) was also promising, as by far most of the analysed reports used solvents recommended in the OECD guideline, i.e. AOO, di-methyl formamide (DMF) and polypropylene glycol, even though the application of the latter is discouraged by the authors of the LLNA (Basketter et al., 2001). Acetone is not included in the OECD guideline, but it is one of the vehicles used in the validation of the LLNA (NTP, 1999); incidentally the use of acetone is generally discouraged for analytical purposes, as it is highly volatile and preparing a solution at a defined concentration is practically impossible. In just one example, a very unusual solvent is applied (1\% Pluronic L92 $\left.{ }^{\circledR}, \mathrm{BASF}\right)$. The OECD guideline specifies the possibility of 
using the commercial formulation in which the test substance is marketed, even though "particular care should be taken to ensure that hydrophilic materials are incorporated into a vehicle system, which wets the skin and does not immediately run off." No justification of the choice is provided in the study.

\section{Tab. 6: Selection of vehicles for testing}

\begin{tabular}{|l|l|}
\hline $\begin{array}{l}\text { Selection of vehicle for tested } \\
\text { substance }\end{array}$ & Number of studies \\
\hline AOO & 10 \\
\hline Propylene glycol & 5 \\
\hline DMF & 2 \\
\hline Acetone & 2 \\
\hline $1 \%$ Pluronic L92 & (BASF) \\
\hline
\end{tabular}

\section{Animal number}

Another interesting issue, especially from an animal welfare perspective, is the number of animals used per test (Tab. 7). The 2002 edition of OECD TG 429 requires a minimum of four animals per group with a minimum of three concentrations of the tested substance. Considering that the negative control also must be included, the minimum number of animals per study is 16 , if the testing lab can demonstrate historical data for a positive control. In practice, the present assessment demonstrates that most of the studies use groups of five mice and that the positive control is very often performed parallel to testing the substance. This means that the average number of mice per tested substances is 25 , not 23 as previously reported (van der Jagt et al., 2004; Rovida and Hartung, 2009). The average number of mice per tested chemical is 25 in the 20 examples considered here (Tab. 7). In four cases, six animals per group were used, and in only one case four groups were used. Compared with the full study reports, the animal number will still be underestimated, as pre-screen test animals and "reserve" animals are not mentioned (cp. Tab. 1). These animals are probably not used to calculate the final response, so they are not reported in IUCLID 5.

The new version of OECD TG 429 includes the option of applying a reduced LLNA test that uses only one concentration of the testing substance and therefore a much smaller number of

\section{Tab. 7: Number of mice per test}

\begin{tabular}{|l|l|l|l|c|}
\hline $\begin{array}{l}\text { Number of } \\
\text { mice } \\
\text { per group }\end{array}$ & $\begin{array}{l}\text { Number of } \\
\text { groups } \\
\text { per study }\end{array}$ & $\begin{array}{l}\text { Number of } \\
\text { mice } \\
\text { per study }\end{array}$ & $\begin{array}{l}\text { Number of } \\
\text { studies }\end{array}$ & Total \\
\hline 4 & 5 & 20 & 3 & 60 \\
\hline 5 & 5 & 25 & 12 & 300 \\
\hline 5 & 4 & 20 & 1 & 20 \\
\hline 6 & 5 & 30 & 4 & 120 \\
\hline Total & & & $\mathbf{5 0 0}$ \\
\hline Average number of mice per study & $\mathbf{2 5}$ \\
\hline
\end{tabular}

animals. This was not done in any of the analysed examples, as probably all of them were performed before July 2010 .

\section{Conclusions}

An early analysis of the registration dossiers submitted to ECHA shows that while the major structure of the main procedures described in the relevant OECD test guidelines are respected, little attention is paid to details that may be peculiar to the specific tested substance. The impression is that testing laboratories show the right attitude following guidelines on animal treatment, but do not consider the chemistry of the substance and disregard any option of reducing the number of animals used per test substance. It appears that a standard protocol is followed with no efforts to tailor it to specific needs.

It further appears that although alternative strategies may be taken into consideration to exploit existing data, they are rarely considered when new tests are required.

Major deviations from the OECD test guidelines are not always explained and/or justified, which may lead to a degree of uncertainty regarding the final results.

It remains to appeal to the actors of the REACH registration process and those who perform experimental toxicological tests to adhere to the following advice: For ethical reasons, in vivo tests should be avoided as much as possible. Each new test should be thoughtfully and meticulously planned to minimise the number of animals while maximising the amount of information acquired. The latter is accomplished by strictly following the guidelines and by extensively reporting the results. This is the only way to accomplish a general standardisation of the procedure that allows a very effective comparison of results obtained in different laboratories on a variety of chemicals. It is also the only way to avoid the need to repeat an experiment. In the long run, high quality data allows more extensive use in read-across and thus a decrease in the number of tests required in the future. A study protocol that is weakly designed leads to poor scientific results and the useless sacrifice of animals.

\section{References}

Basketter, D. A., Gerberick, G. F. and Kimber, I. (2001). Skin sensitisation, vehicle effects and the local lymph node assay. Food Chem. Toxicol. 39 (6), 621-627.

Basketter, D. A., Cockshott, A., Corsini, E. et al. (2008). An evaluation of performance standards and non-radioactive endpoints for the local lymph node assay. ATLA 36, 243-257.

Basketter, D. A. and Kimber, I. (2009). Skin sensitization, false positives and false negatives: experience with guinea pig assays. J. Appl. Toxicol. 30, 381-386.

Basketter, D. A., McFadden, J. F., Gerberick, F. et al. (2009). Nothing is perfect, not even the local lymph node assay: a commentary and the implications for REACH. Contact Dermatitis 60, 65-69.

Burns, T., Strickland, J., Salicru, E. et al. (2010). Comparison of responses in the murine local lymph node assay (LLNA) between CBA and BALB/c mouse strains. Poster presented at the 
Annual Meeting of the Society of Toxicology.

Cockshott, A., Evans, P., Ryan, C. A. et al. (2006). The local lymph node assay in practice: a current regulatory perspective. Hum. Exp. Toxicol. 25(7), 387-394.

EC - European Commission (2006). Regulation (EC) of No $1907 / 2006$ of the European parliament and of the council 18 December 2006 concerning the Registration, Evaluation, Authorisation and Restriction of Chemicals (REACH), establishing a European Chemicals Agency, amending Directive 1999/45/EC and repealing Council Regulation (EEC) No 793/93 and Commission Regulation (EC) No 1488/94 as well as Council Directive 76/769/EEC and Commission Directives 91/155/EEC, 93/67/EEC, 93/105/EC and 2000/21/EC

EC (2008a). Regulation (EC) No 440/2008 of 30 May 2008 laying down test methods pursuant to Regulation (EC) No 1907/2006 of the European Parliament and of the Council on the Registration, Evaluation, Authorisation and Restriction of Chemicals (REACH).

EC (2008b). Regulation (EC) No 1272/2008 of the European Parliament and of the Council of 16 December 2008 on classification, labelling and packaging of substances and mixtures, amending and repealing Directives 67/548/EEC and 1999/45/ EC, and amending Regulation (EC) No 1907/2006

EC (2011). Commission Regulation (EU) No 286/2011 of 10 March 2011 amending, for the purposes of its adaptation to technical and scientific progress, Regulation (EC) No 1272/2008 of the European Parliament and of the Council on classification, labelling and packaging of substances and mixtures.

ECHA (2008). Guidance on information requirements and chemical safety assessment. Chapter R.7a: Endpoint specific guidance.http://guidance.echa.europa.eu/docs/guidance_document/ (accessed 23.02.2011).

ECHA Practical Guide 3. How to report robust study summaries. ECHA-10-B-06-EN, 24 ${ }^{\text {th }}$ March 2010. http://echa.europa.eu/ publications_en.asp (accessed 23.02.2011).

EPA (2003). OPPTS 270.2600 Skin Sensitisation. EPA 712-C03-197, http://www.epa.gov/ocspp/pubs/frs/publications/Test_ Guidelines/series870.htm

Gerberick, G. F., Ryan, C. A., Dearman, R. J. and Kimber, I. (2007). Local lymph node assay (LLNA) for detection of sensitization capacity of chemicals. Methods 41, 54-60.

Hoffmann, S. and Hartung, T. (2006). Toward an evidence-based toxicology. Hum. Exp. Toxicol. 25(9), 497-513.

ICCVAM (2009). Draft ICCVAM test method recommendations updated assessment of the validity of the LLNA for testing pesticide formulations and other products, metals, and substances in aqueous solutions. http://iccvam.niehs.nih.gov/methods/immunotox/LLNA-app/recs.pdf (accessed 23.02.2011).

Jowsey, I. R., Clapp, C. J., Safford, B. et al. (2008). The impact of vehicle on the relative potency of skin sensitizing chemicals in the local lymph node assay. Cutan. Ocul. Toxicol. 27, 67-75.

Kimber, I. and Basketter, D. A. (1992). The murine local lymph node assay; collaborative studies and new directions: A commentary. Food Chem. Toxicol. 30, 165-169.

NTP (1999). The murine local lymph node assay: A test method for assessing the allergic contact dermatitis potential of chemi- cals/compounds. The results of an independent peer review evaluation coordinated by the Interagency Coordinating Committee on the Validation of Alternative Methods (ICCVAM) and the National Toxicology Program Center for the Evaluation of Alternative Toxicological Methods (NICEATM). NIH Publication No. 99-4494. http://iccvam.niehs.nih.gov/methods/ immunotox/llna_PeerPanel.htm (accessed 23.02.2011).

NTP (2005). Descriptions of NTP study types: Hypersensitivity test methods. http://ntp.niehs.nih.gov

OECD TG 406. Skin sensitisation. OECD Guideline For Testing Of Chemicals. (Adopted 17.07.1992).

OECDTG 429. Skin sensitisation: Local lymph node assay. OECD Guideline For Testing Of Chemicals. (Adopted 24.04.2002).

OECD TG 429. Skin Sensitisation: Local lymph node assay. OECD Guideline For Testing Of Chemicals. (Adopted 22.07.2010).

OECD TG 428. Skin absorption: In vitro method. OECD Guideline For Testing Of Chemicals. (Adopted 13.04.2004).

OECD TG 442a. Skin sensitisation: Local lymph node assay: DA. OECD Guideline For Testing Of Chemicals. (Adopted 22.07.2010).

OECD TG 442b. Skin sensitisation: Local lymph node assay: BrdU-ELISA. OECD Guideline For Testing Of Chemicals. (Adopted 22.07.2010).

Rovida, C. and Hartung, T. (2009). Re-evaluation of animal numbers and costs for in vivo tests to accomplish REACH legislation requirements for chemicals. ALTEX 26, 187-202.

Rovida, C. (2010). Food for thought ... why no new in vitro tests will be done for REACH by registrants. ALTEX 27(3), 175183.

van der Jagt, K., Munn, S., Tørsløv, J. and de Bruijn, J. (2004). Alternative approaches can reduce the use of test animals under reach. Addendum to the report: Assessment of additional testing needs under REACH effects of (Q)SARS, risk based testing and voluntary industry initiatives. JRC Report EUR 21405 EN.

Woolhiser, M., Munson, A. and Meade, J. (2000). Comparison of mouse strains using the local lymph node assay. Toxicology $146(2-3), 221-227$.

\section{Acknowledgments}

I wish to thank Prof. Dr. Thomas Hartung and CAAT staff for critically reading this manuscript. I am also grateful to Prof. Kimber and Dr. Basketter who helped me understand the benefits of the LLNA. Prof. Kimber suggested the idea of writing this contribution.

\section{Correspondence to}

Costanza Rovida

Center for Alternatives to Animal Testing - Europe

(CAAT-Europe)

University of Konstanz, POB 600

78457 Konstanz

Germany

e-mail: costanza.rovida@chimici.it 